\title{
Analysis of the pressure fields in a swirling annular jet flow
}

\author{
M. Percin ${ }^{1,2} \cdot$ M. Vanierschot ${ }^{3}$ B. W. van Oudheusden ${ }^{1}$
}

Received: 7 February 2017 / Revised: 17 September 2017 / Accepted: 19 September 2017 / Published online: 8 November 2017

(C) The Author(s) 2017. This article is an open access publication

\begin{abstract}
In this paper, we investigate the flow structures and pressure fields of a free annular swirling jet flow undergoing vortex breakdown. The flow field is analyzed by means of time-resolved tomographic particle image velocimetry measurements, which enable the reconstruction of the three-dimensional time-resolved pressure fields using the governing flow equations. Both time-averaged and instantaneous flow structures are discussed, including a characterization of the first- and second-order statistical moments. A Reynolds decomposition of the flow field shows that the time-averaged flow is axisymmetric with regions of high anisotropic Reynolds stresses. Two recirculation zones exist that are surrounded by regions of very intense mixing. Notwithstanding the axisymmetric nature of the time-averaged flow, a non-axisymmetric structure of the instantaneous flow is revealed, comprising a central vortex core which breaks up into a precessing vortex core. The winding sense of this helical structure is opposite to the swirl direction and it is wrapped around the vortex breakdown bubble. It precesses around the central axis of the flow at a frequency corresponding to a Strouhal number of 0.27 . The precessing vortex core is associated with a low-pressure region along the central axis of the jet and the maximum pressure fluctuations occur upstream of the vortex breakdown location, where the
\end{abstract}

M. Percin

m.percin@tudelft.nl

1 Department of Aerospace Engineering, Delft University of Technology, Kluyverweg 1, 2629 HS, Delft, The Netherlands

2 Department of Aerospace Engineering, Middle East Technical University, Ankara, Turkey

3 KU Leuven, Mechanical Engineering Technology TC, Campus Group T Leuven, A. Vesaliusstraat 13, 3000 Leuven, Belgium azimuthal velocity component also reaches peak values as a result of the inward motion of the fluid and the conservation of angular momentum. The POD analysis of the pressure fields suggests that the precessing helical vortex formation is the dominant coherent structure in the instantaneous flow.

Keywords Swirling annular jet - Tomographic particle image velocimetry $\cdot$ Pressure reconstruction · Vortex breakdown

\section{Introduction}

Annular jet flows are of practical interest in view of their occurrence in many industrial applications in the context of bluffbody combustors (Gupta et al. 1984). Due to flow separation in the immediate wake of the bluff-body, a region of subambient pressure is generated. This central recirculation zone (CRZ) is favorable in terms of promoting flow mixing and flame stabilization (Beér and Chigier 1972). Moreover, the central bluff-body can be used as a fuel injection device in non- or partially premixed combustion either using cross-flow (Dugué and Weber 1992a, b) or co-flow injection (Al-Abdeli and Masri 2004; García-Villalba and Fröhlich 2006; Warda et al. 1999).

In addition to the aforementioned CRZ, annular jet flows feature different complex flow characteristics despite their simple geometry: an outer (between the jet and the environment) and inner (between the jet and the central recirculation region) shear layer, which are both characterized by strong anisotropic turbulence (Vanierschot et al. 2014). The complexity of the flow is further enhanced when introducing swirl which leads to the formation of large zones of recirculation and large-scale instabilities at certain swirl numbers, such as vortex breakdown or a precessing vortex core (PVC) (Vanierschot and Van den Bulck 2008a; Lucca-Negro and O'Doherty 
2001). These large coherent structures have been well studied for swirling circular jet flows (Panda and McLaughlin 1994; Billant et al. 1998; Al-Abdeli and Masri 2004; Cala et al. 2006; Oberleithner et al. 2011; Martinelli et al. 2012; Litvinov et al. 2013; Markovich et al. 2014; Oberleithner et al. 2014). However, for the case of annular jet flows, much remains to be resolved, especially regarding the interaction between the instabilities and the CRZ. Many studies on annular jet geometries have considered reacting flows, as their main application is in swirl combustors. Studies have shown that these largescale precessing structures found in cold flows are also present in combustion. The PVC has a strong influence on the flame shape and position, pollutant formation and resonance phenomena (Chterev et al. 2014; Reichel et al. 2015; Oberleithner et al. 2015; Ghani et al. 2015; Chterev et al. 2017). Sheen et al. (1996) performed one of the earlier studies for cold flows in this respect, in which they investigated the recirculation zones in both confined and unconfined annular swirling jet flows by changing the Reynolds number (Re) and swirl numbers. They used smoke flow visualizations to inspect the dynamic flow features in the recirculation zone of the bluff body, which are classified into seven typical patterns. They observed that a vortex breakdown bubble is formed at an intermediate swirl number, which moves upstream as the swirl number increases. The CRZ and the breakdown bubble merge to form a single recirculation zone at a sufficiently high swirl. Despite the descriptive flow visualization results, only a limited amount of quantitative data are presented in this paper with a lack of the analysis of turbulence characteristics or time-resolved information of the dynamic flow features. Although there have been a number of numerical studies focusing on the recirculation zones of annular swirling jet flows (García-Villalba and Fröhlich 2006; García-Villalba et al. 2006; Wegner et al. 2004), the first experimental studies to obtain flow field information resolved in both time and space were performed by (Vanierschot and Van den Bulck 2008a) by conducting timeresolved stereoscopic particle image velocimetry (StereoPIV) measurements in the central plane of the annular jet. In subsequent works, they investigated the dynamics of the precessing vortex core (Vanierschot and Van den Bulck 2011; Vanierschot et al. 2014) and calculated the mean pressure field in the initial merging zone of the swirling jet (Vanierschot and Van den Bulck 2008b). However, these studies are limited due to the planar measurement of the three-dimensional swirling jet flow fields.

In this regard, the specific aim of the current study is, therefore, to investigate the spatial and temporal characteristics of the three-dimensional flow fields in a swirling annular jet flow, employing time-resolved tomographic particle image velocimetry (tomographic-PIV) measurements (Elsinga et al. 2006; Scarano 2012). The image acquisition was performed in two modes, i.e., a low frequency doubleframe mode and a high frequency single-frame mode, to enable converged statistical analysis and visualization of the time-series phenomenon, respectively. The volumetric velocity fields were also used to calculate the time-averaged and instantaneous pressure fields by employing the flow governing (Navier-Stokes) equations (van Oudheusden 2013). In this respect, by providing three-dimensional experimental pressure field data for the annular swirling jet flow, the present study aims at improving the understanding of the inherent flow characteristics and may serve as a benchmark case for further experimental and numerical investigations.

\section{Experimental setup and processing methods}

\subsection{Experimental setup}

The experiments were conducted in a water facility at the Aerodynamic Laboratories of Delft University of Technology. An annular jet orifice with an inner diameter $D_{i}=18 \mathrm{~mm}$ and an outer diameter $D_{o}=27 \mathrm{~mm}$ was installed at the bottom wall of the octagonal water tank (600 $\mathrm{mm}$ of diameter and $800 \mathrm{~mm}$ of height), which is made of Plexiglass to enable full optical access for illumination and tomographic imaging (Percin and Van Oudheusden 2015). The symmetry axis of the jet is aligned with the (vertical) $y$-axis in the measurement coordinate system with the origin located at the exit of the inner tube (see Fig. 1). The experiments were performed at a Reynolds number of 8500 based on the hydraulic diameter of the annular jet $\left(D_{h}=9 \mathrm{~mm}\right)$ and the mean axial velocity of the jet $\left(U_{0}=0.94 \mathrm{~m} / \mathrm{s}\right)$. The flow in the system was driven by a pump that was submerged in a reservoir containing water

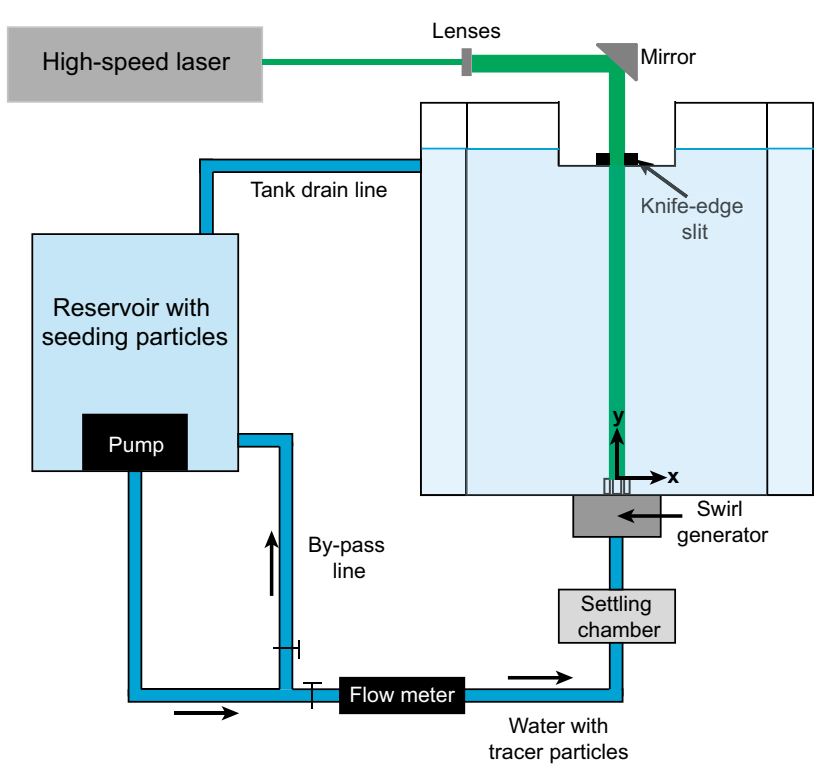

Fig. 1 A sketch of the experimental setup 
mixed with seeding particles. The swirl was generated by means of a block swirl generator, which consists of 12 guide vanes that can be adjusted to change the swirl strength. A detailed description of the swirler geometry can be found in Dugué and Weber (1992a) and Vanierschot and Van den Bulck (2008a). In this study, the swirl number is defined based on the axial flux of tangential and axial momentum as:

$S=\frac{\int_{R_{i}}^{R_{o}} 2 \pi \rho \overline{U_{y} W} r^{2} d r}{R_{o} \int_{R_{i}}^{R_{o}} 2 \pi\left(\rho{\overline{U_{y}}}^{2}\right) r d r}$

where $\overline{U_{y}}$ and $\bar{W}$ are the mean axial and azimuthal velocity components, respectively. The swirl number is calculated as 0.4 in the cross-flow measurement plane that is nearest to the jet exit.

\subsection{Tomographic particle image velocimetry}

Neutrally buoyant polyamide spherical particles of $56 \mu$ $\mathrm{m}$ mean diameter were employed as tracer particles at a concentration of 0.65 particles $/ \mathrm{mm}^{3}$. The flow was illuminated by a double-pulse Nd:YLF laser (Quantronix Darwin Duo, $2 \times 25 \mathrm{~mJ} /$ pulse at $1 \mathrm{kHz}$ ) at a wavelength of $527 \mathrm{~nm}$ (Fig. 2a). The light scattered by the particles was recorded by a tomographic system composed of four LaVision HighSpeedStar 6 CMOS cameras $(1024 \times 1024$ pixels, $5400 \mathrm{frames} / \mathrm{s}$, pixel pitch of $20 \mu \mathrm{m})$. Each camera was equipped with a Nikon $105 \mathrm{~mm}$ focal objective with a numerical aperture $f \#=32$ to allow focused imaging of the illuminated particles. The cameras were linearly arranged in a horizontal plane (Fig. 2a) with an aperture angle of $90^{\circ}$ (Fig. 2b). A pair of diverging and converging spherical lenses was used to form a cylindrical volume with a diameter of $3.6 D_{h}$ and a height of $5.3 D_{h}$. The measurements were performed in this volume at a digital resolution of 21.6 pix$\mathrm{els} / \mathrm{mm}$. The choice of a cylindrical measurement volume eliminated the need for a lens-tilt mechanism to comply with the Scheimpflug condition. Moreover, the cylindrical volume brings about a more favorable condition for the accurate reconstruction since the particle image density does not vary with the viewing angle along the azimuth and decreases when moving toward the periphery of the jet. The average particle image density is approximately 0.045 particles per pixel (ppp). The images were captured with two recording modes: (1) a double-frame mode at a low recording frequency of $50 \mathrm{~Hz}$ to allow a converged statistical analysis by using statistical independent samples and capturing the flow for a longer period of time; (2) a single-frame mode at a high recording frequency of $2.5 \mathrm{kHz}$ to enable the visualization of time-series phenomena. In the former case, a total of 2728 images were captured over a duration of $48.3 \mathrm{~s}$, whereas for
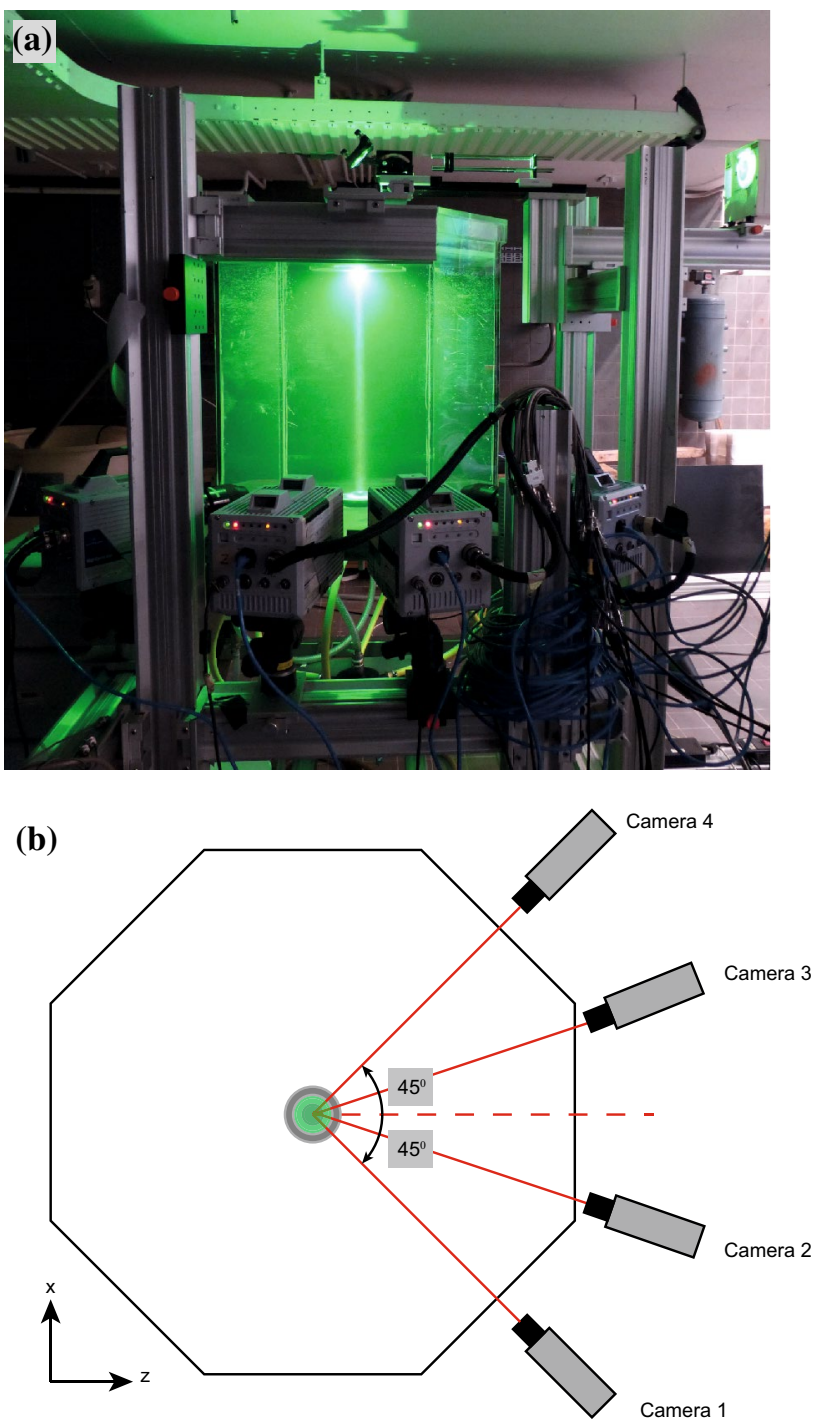

Fig. 2 a Tomographic-PIV setup, b sketch of the top view of the experimental setup with camera arrangement

the latter the measurement duration was limited to approximately $2.2 \mathrm{~s}$, collecting 5456 images.

Image pre-processing, volume calibration, self-calibration, reconstruction and three-dimensional cross-correlation-based interrogation were performed in LaVision DaVis 8.1.6. The measurement volume was calibrated by scanning a calibration target through the measurement volume. A third-order polynomial was fitted as the mapping function that provides the relation between the image coordinates and the physical coordinates for each camera. The initial calibration was refined by means of the volume self-calibration technique (Wieneke 2008), resulting in a misalignment of less than 0.05 pixels. The raw images were pre-processed with background intensity removal and particle intensity normalization. The tomographic reconstruction was performed by using MLOS initialization (Atkinson and Soria 2009) and 10 CSMART iterations with 
Gaussian smoothing after each iteration. The particle images were then interrogated using an iterative, multigrid correlation with a window deformation procedure. Interrogation volumes of final size $48 \times 48 \times 48$ voxels with an overlap factor of $75 \%$ yielded a vector spacing of $0.56 \mathrm{~mm}$ in each direction. Spurious velocity vectors are removed by the universal median test (Westerweel and Scarano 2005) and a second-order polynomial regression in time and space was applied to reduce the noise in the resultant vector fields.

\subsection{Calculation of the pressure fields}

The three-dimensional velocity fields obtained by means of the tomographic-PIV measurements are used for the calculation of the instantaneous and time-averaged pressure fields of the annular swirling jet flow. For the calculation of the instantaneous pressure fields, the pressure gradients are computed by means of the momentum equation as follows:

$\boldsymbol{\nabla} P=-\rho \frac{\mathrm{D} \mathbf{U}}{\mathrm{D} t}+\mu \nabla^{2} \mathbf{U}$,

where $\mathrm{DU} / \mathrm{D} t$ stands for the material acceleration, which was evaluated in a Lagrangian perspective by means of least square fit of the velocities along a reconstructed particle trajectory (Pröbsting et al. 2013). The selected stencil size of $4 \Delta t$ was based on the relatively high correlation of the velocity fields within this time range. Then, for the calculation of the instantaneous pressure field, the corresponding Poisson problem was solved for the complete flow field by assigning Dirichlet boundary conditions (a constant ambient pressure) at the lateral surfaces and Neumann boundary conditions at the upper and lower surfaces of the cylindrical measurement domain (see Fig. 3).

The instantaneous pressure fields have been further analyzed by the proper orthogonal decomposition technique (POD) following the method of snapshots (Sirovich 1987) to determine the energetic and coherent structures contributing to the pressure fluctuations. POD provides ranking of the orthogonal structures and to capture their temporal dynamics, projection of the full data sequence onto the finite POD basis is performed yielding the time coefficients associated with each individual POD mode.

The time-averaged pressure field is calculated by averaging these instantaneous pressure fields, as well as from Reynolds averaging of the momentum equation (as indicated by the overbar) which allows the mean pressure gradient to be calculated from the velocity field statistics, as follows (van Oudheusden 2013):

$\boldsymbol{\nabla} \bar{P}=-\rho(\overline{\mathbf{U}} \cdot \boldsymbol{\nabla}) \overline{\mathbf{U}}-\rho \boldsymbol{\nabla} \cdot(\overline{\mathbf{u u}})+\mu \nabla^{2} \overline{\mathbf{U}}$

where $\overline{\mathbf{U}}$ stands for the time-averaged velocity and $\mathbf{u}$ for the fluctuating component of the velocity. In this approach, the

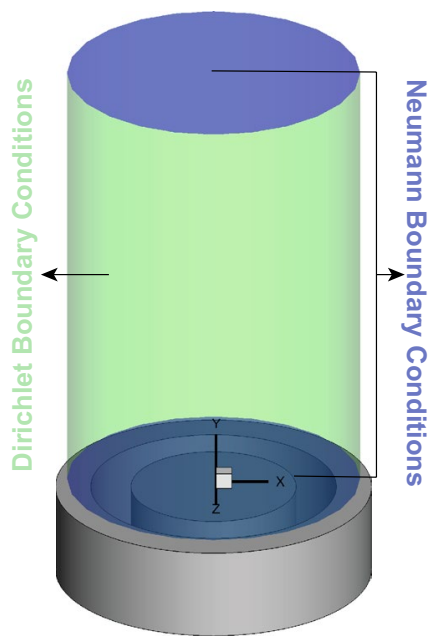

Fig. 3 Configuration of boundary conditions for the solution of the Poisson problem (green Dirichlet boundary conditions, blue Neumann boundary conditions)

Reynolds stress terms are to be included in the momentum equation, whereas the time-derivative term is no longer required in the calculation of the pressure gradients. These terms are acquired from the ensemble of 2728 statistically independent individual velocity fields and the corresponding Poisson problem was solved with the boundary condition settings identical to the instantaneous pressure calculations. Although the convective term (the first term on the righthand side of Eq. 3) was found to be dominant, all terms were included in the calculation of pressure gradients for the sake of completeness.

\subsection{Accuracy of PIV measurements}

The accuracy of the three-dimensional experimental data can be evaluated in relation to two different aspects: the accuracy of the instantaneous velocity fields and the errors affecting the statistical quantities. The former part contains random and bias error components, which are introduced into the instantaneous data by several sources regarding the experimental instruments, acquisition and processing techniques. To evaluate the random error component, the spatial distribution of velocity divergence is utilized. In the incompressible flow regime, the divergence of both the mean $\overline{\mathbf{U}}$ and fluctuating $\mathbf{u}$ velocity components should be zero in the absence of measurement error (Scarano et al. 2006; Atkinson et al. 2011). Therefore, under the assumption of uniform random error distribution in each direction, the relation between the error in the velocity gradient, which is calculated using a second-order central difference scheme, and the random velocity error $\delta(u)$ is obtained as follows (Atkinson et al. 2011): 
$\delta\left(\frac{\partial u_{i}}{\partial x_{i}}\right)=\sqrt{\frac{3}{2 d_{v}^{2}}} \delta(u)$

where $d_{v}$ is the vector spacing. Using the observed RMS of the fluctuating divergence, the random velocity error for the present experiments can be estimated. The RMS of the fluctuating divergence, as averaged over the measurement region, returned 0.03 pixels/pixel, which is of the same order of magnitude as 0.02 pixels/pixel reported by Scarano et al. (2006) and 0.05 pixels/pixel reported by Atkinson et al. (2011). This corresponds to 0.3 pixels velocity random error for the time interval between two consecutive images (i.e., $400 \mu \mathrm{s})$. In physical units, this returns $\delta(u) / U_{0}=3.6 \%$ for the present experiments. Regarding the velocity gradients, analysis of the fluctuating divergence yields approximately $8 \%$ uncertainty in the measured range of vorticity for the time-averaged velocity field.

In the statistical analysis of flow fields obtained with PIV, two kind of errors affect the calculation of the flow field variables. The first error is the convergence error resulting from the calculation of statistical properties from a finite number of samples. In addition to this sampling error, a further error arises from the spatial resolution of the PIV measurements. If the spatial resolution of the measurements is the interrogation window size $\Delta$, the maximum wavenumber in the turbulent spectrum that can be measured is $k_{\max }=\pi / \Delta$ (Willert and Gharib 1991). Due to low-pass filtering in the vector processing, the measured second-order statistics are attenuated compared to the real ones when the size of the interrogation window is too large. In the following discussion, both errors are quantified and it is shown that the results in Sect. 3.1 are sufficiently accurate to serve as a database for the validation of numerical codes.

For the statistical analysis of the flow, $N=2728$ statistically independent samples are taken to calculate the first- and second-order moments of the flow. The statistical uncertainty estimates of the mean flow and the Reynolds stresses associated with the sampling of the phenomenon (under the assumption that the samples can be considered uncorrelated and follow a normal distribution of standard deviation) can be calculated as (Benedict and Gould 1996; Sciacchitano and Wieneke 2016):

$$
\begin{aligned}
& \delta_{\overline{U_{i}}}=Z_{\alpha / 2} \sqrt{\frac{\overline{u_{i}^{2}}}{N}}, \\
& \delta_{\overline{u_{i}^{2}}}=Z_{\alpha / 2} \sqrt{\frac{2\left(\overline{u_{i}^{2}}\right)^{2}}{N}}, \\
& \delta_{\overline{u_{i} u_{j}}}=Z_{\alpha / 2} \sqrt{\overline{u_{i}^{2}} \overline{u_{j}^{2}}\left(\frac{1+R_{u_{i} u_{j}}^{2}}{N}\right)} \quad(i \neq j),
\end{aligned}
$$

where $Z_{\alpha / 2}=1.96$ for a $95 \%$ confidence interval and $R_{u_{i} u_{j}}$ is the correlation coefficient between the two fluctuating components, which reads:

$$
R_{u_{i} u_{j}}=\frac{\overline{u_{i} u_{j}}}{\sqrt{\overline{\overline{u_{i}^{2}}} \overline{\overline{u_{j}^{2}}}} .}
$$

The statistical uncertainty estimates for the mean flow and Reynolds stress terms are summarized in Table 1. It should be noted that mean uncertainty estimates averaged over the entire measurement volume are presented in both absolute and normalized values.

To estimate the error introduced by the finite interrogation window size, the procedure of Alekseenko et al. (2007) is followed. This procedure calculates the Kolmogorov and integral length scales of the flow and provides estimates of the error on the second-order statistic based on the ratio between the window size and these length scales. First, the uncorrected turbulent dissipation rate $\epsilon$ is calculated from the measurements as:

$$
\begin{aligned}
\epsilon_{\text {meas }}= & \nu\left\langle 2\left(\frac{\partial u_{x}}{\partial x}\right)^{2}+2\left(\frac{\partial u_{y}}{\partial y}\right)^{2}+2\left(\frac{\partial u_{z}}{\partial z}\right)^{2}\right. \\
& +\left(\frac{\partial u_{x}}{\partial z}+\frac{\partial u_{z}}{\partial x}\right)^{2}+\left(\frac{\partial u_{y}}{\partial x}+\frac{\partial u_{x}}{\partial y}\right)^{2} \\
& \left.+\left(\frac{\partial u_{z}}{\partial y}+\frac{\partial u_{y}}{\partial z}\right)^{2}\right\rangle
\end{aligned}
$$

Table 1 The statistical uncertainty estimates for the mean flow (mean values calculated in the entire cylindrical measurement volume with mean of the normalized values in brackets) and the Reynolds stresses (mean non-dimensional values normalized by the reference velocity and Reynolds shear stresses in brackets)

\begin{tabular}{ll}
\hline Mean flow & $\mathrm{m} / \mathrm{s}\left(\times \mathbf{1 0 0} / \mathbf{U}_{\mathbf{0}}\right)_{\text {mean }}$ \\
$\delta_{\overline{U_{x}}}$ & $0.0045(\mathbf{0 . 4 8})$ \\
$\delta_{\overline{U_{y}}}$ & $0.0053(\mathbf{0 . 5 6})$ \\
$\delta_{\overline{U_{z}}}$ & $0.0047(\mathbf{0 . 5})$ \\
$\delta_{|\bar{U}|}$ & $0.0085(\mathbf{0 . 9})$ \\
Reynolds normal stress & $\times 100 / U_{0}^{2}\left(\times \mathbf{1 0 0} /\left(\overline{\mathbf{u}_{\mathbf{i}}}\right)\right)_{\text {mean }}$ \\
$\delta_{\overline{u_{x}^{2}}}$ & $0.1410(\mathbf{7 . 5})$ \\
$\delta_{\overline{u_{y}^{2}}}$ & $0.1875(\mathbf{7 . 5})$ \\
$\delta_{\overline{u_{z}^{2}}}$ & $0.1479(\mathbf{7 . 5})$ \\
Reynolds shear stress & $\times 100 / U_{0}^{2}\left(\times \mathbf{1 0 0} /\left(\overline{\mathbf{u}_{\mathbf{i}} \mathbf{u}_{\mathbf{j}}}\right)\right)_{\text {mean }}$ \\
$\delta_{\overline{u_{x} u_{y}}}$ & $0.043(\mathbf{1 2 . 5})$ \\
$\delta_{\overline{u_{x} u_{z}}}$ & $0.037(\mathbf{1 2 . 9})$ \\
$\delta_{\overline{u_{y} u_{z}}}$ & $0.044(\mathbf{1 2 . 3})$ \\
\hline
\end{tabular}


Table 2 Maximum dissipation rate $(\epsilon)$, Kolmogorov $(\eta)$ and integral $(L)$ length scales of the flow compared to the interrogation area size $\Delta$

\begin{tabular}{llllll}
\hline$\Delta[\mathrm{mm}]$ & $\eta[\mathrm{mm}]$ & $\epsilon\left[\mathrm{m}^{2} / \mathrm{s}^{3}\right]$ & $L[\mathrm{~mm}]$ & $\Delta / \eta$ & $\Delta / L$ \\
\hline 1.12 & 0.024 & 3.13 & 2.7 & 46 & 0.42 \\
\hline
\end{tabular}

where \langle\rangle indicates an ensemble averaging operation and $v$ is the fluid kinematic viscosity. This dissipation rate serves as an input for the calculation of the Kolmogorov length scale as $\eta=\left(v^{3} f_{\epsilon} / \epsilon_{\text {meas }}\right)^{1 / 4}$, where $f_{\epsilon}$ is taken to be one at the start of the procedure. From this length scale, the correction factor $f_{\epsilon}$ can be updated as a function of the ratio $\Delta / \eta$. With

(a) $y / D_{o}=0.2$

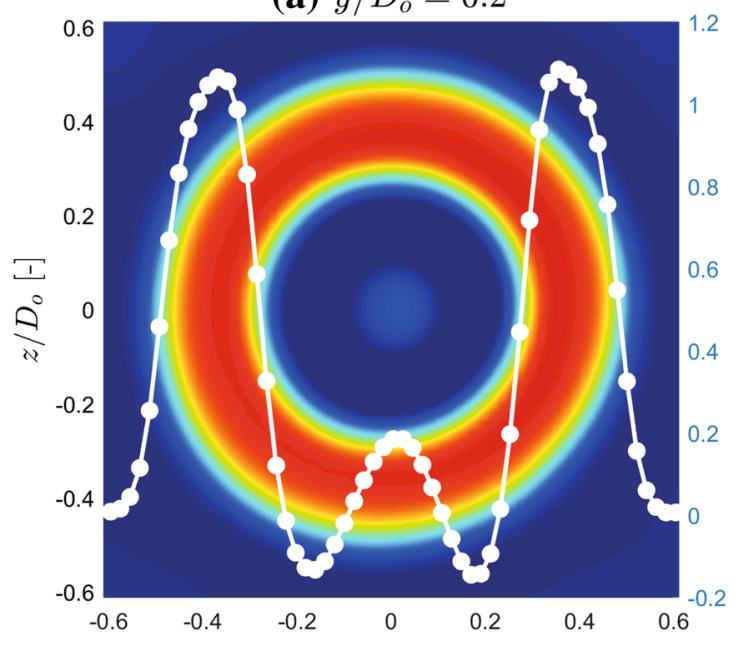

(c) $y / D_{o}=0.8$

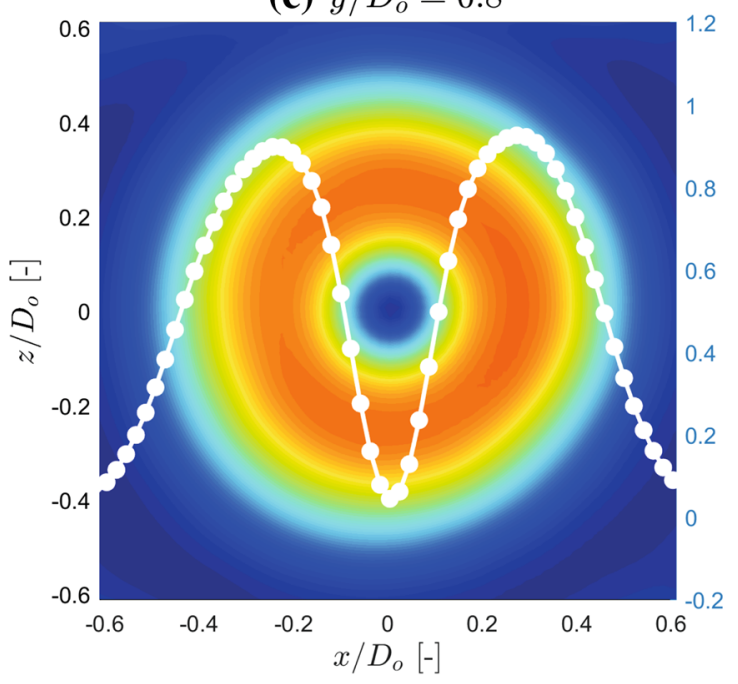

this new correction factor, the Kolmogorov length scale is recalculated and the procedure is repeated until convergence is obtained. For the measurements in this study, the results are summarized in Table 2. The integral length scale $L$ of the flow can be estimated as:

$L=\left(\frac{\left\langle u_{x}^{2}+u_{y}^{2}+u_{z}^{2}\right\rangle}{3}\right)^{\frac{3}{2}} / \epsilon$

Based on the study of Alekseenko et al. (2007), the maximum error for the second-order statistics in the current experiment is around $8 \%$ and the interrogation size $\Delta$ is within the inertial sub-range of the turbulent spectrum.

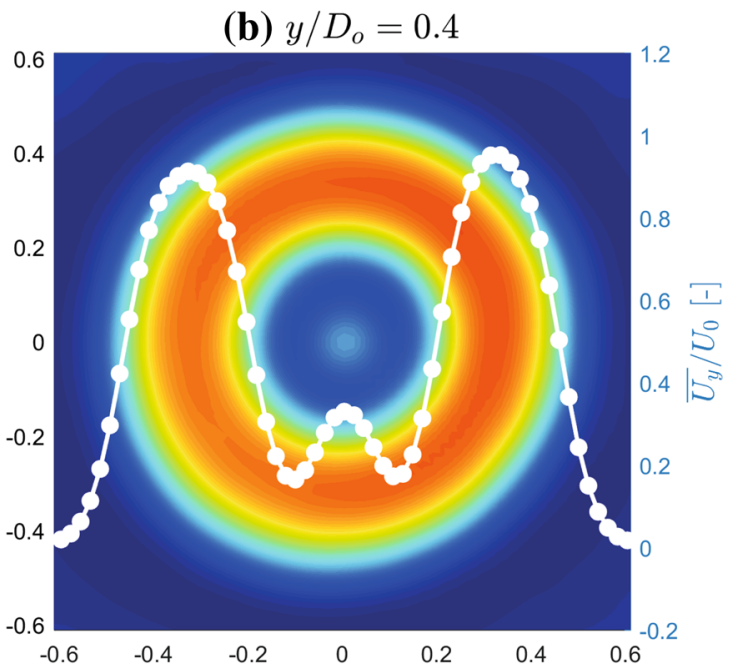

(d) $y / D_{o}=1.2$

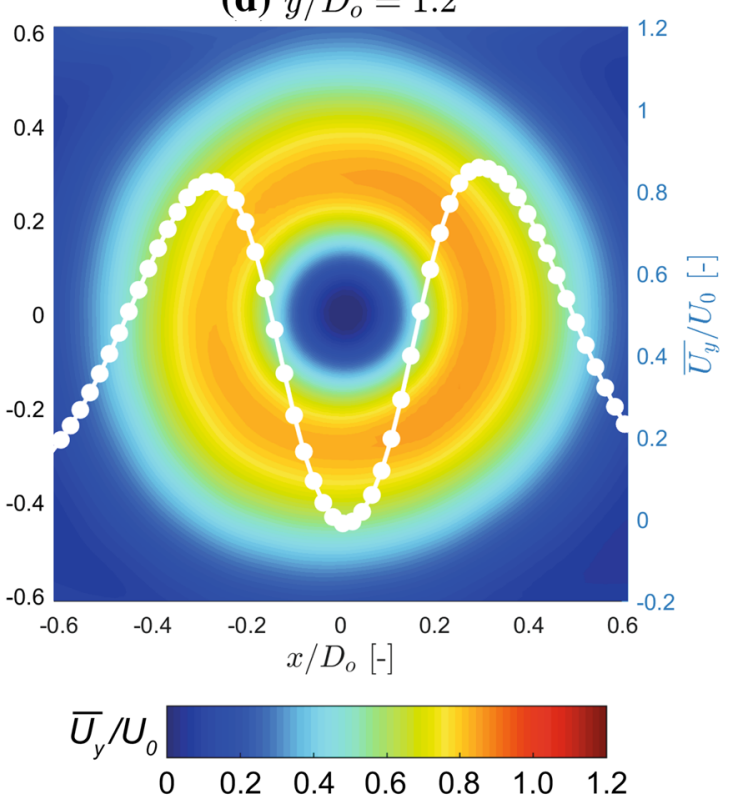

Fig. 4 Time-averaged contours of the axial velocity component $\left(\overline{U_{y}}\right)$ complemented with the $U_{y}$ vs. $x$ variation at $z / D_{0}=0$ location $($ white $)$ plotted in several cross-flow planes $\left(x-z\right.$ plane): a $y / D_{0}=0.2$, b $y / D_{0}=0.4, \mathbf{c} y / D_{0}=0.8$, and $\mathbf{d} y / D_{0}=1.2$ 
(a) $\overline{U_{x}} / U_{0}$

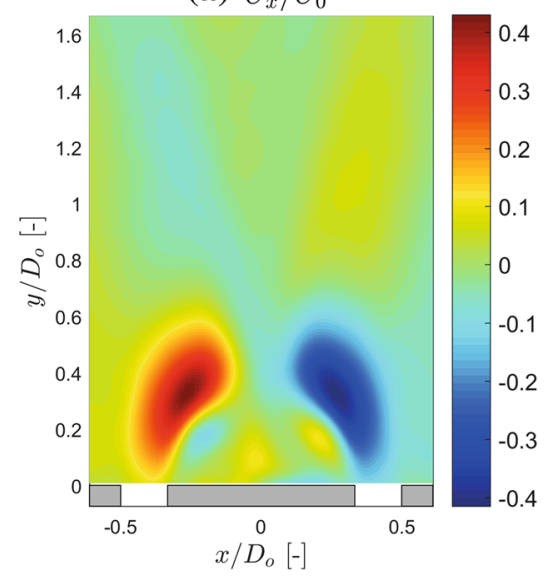

(b) $\overline{U_{y}} / U_{0}$

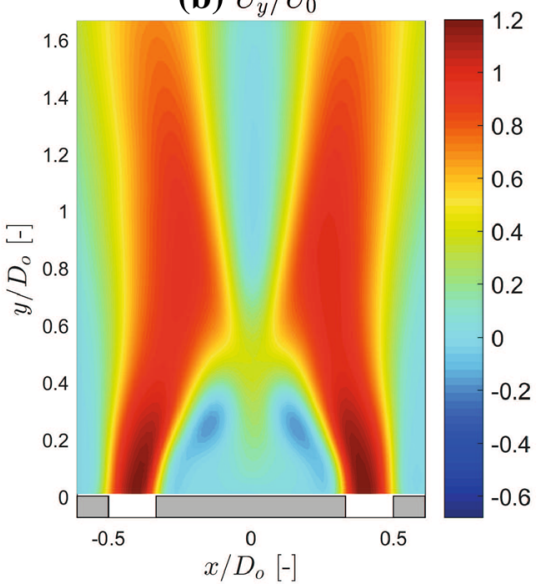

(c) $\overline{U_{z}} / U_{0}$

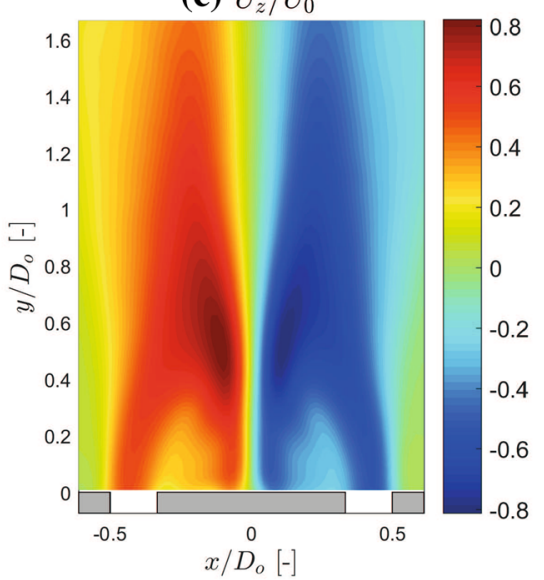

Fig. 5 Time-averaged fields of the velocity components in a $x$ direction $\left(\overline{U_{x}}\right.$, corresponding to the radial velocity component), b $y$ direction $\left(\bar{U}_{y}\right.$, corresponding to the axial velocity component), c $z$ direc-

\section{Results}

\subsection{Time-averaged flow quantities}

The analysis of the time-averaged flow fields applies a Reynolds decomposition, which divides the velocity vector into mean and fluctuating components. The flow field is axisymmetric as shown in Fig. 4 at several cross-flow planes and hence a planar representation is sufficient to document the flow structure. Accordingly, Fig. 5 displays the spatial distribution of the mean velocity components $\overline{U_{x}}, \overline{U_{y}}$ and $\overline{U_{z}}$ in the central plane of the jet $\left(z / D_{0}=0\right)$, which in the context of swirling flows represent the radial, axial and azimuthal velocity. All quantities are scaled with respect to the mean axial jet velocity, $U_{0}$.

As shown in Figure 5b, the axial velocity distribution reveals two prominent regions of backflow, i.e., negative $\overline{U_{y}}$. (a) $d P / d R$

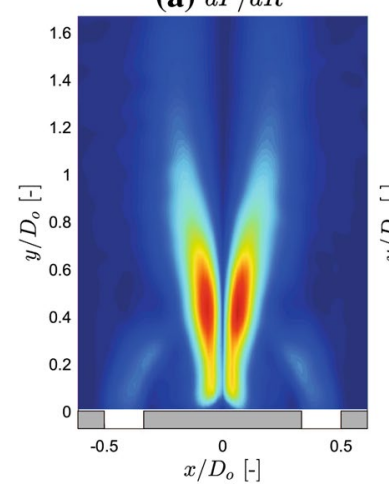

(b) $\rho W^{2} / R$

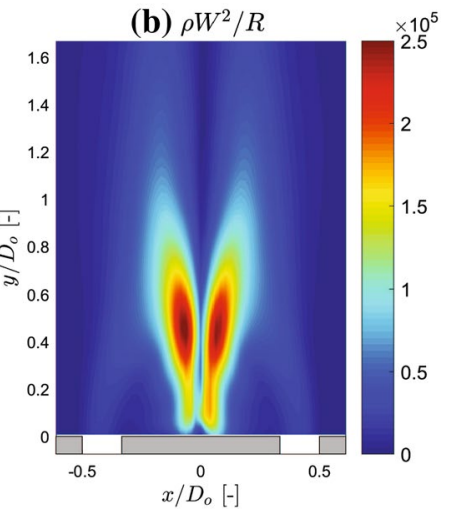

Fig. 6 Time-averaged fields of the a pressure gradient, and $\mathbf{b}$ centrifugal force terms tion $\left(\overline{U_{z}}\right.$, corresponding to the azimuthal velocity component) plotted in the central plane of the jet $(z=0)$

The first region is the CRZ directly behind the central body. In case of non-swirling flow, this recirculation zone is closed at a stagnation point in the flow downstream (Vanierschot and Van den Bulck 2008a). However, swirl induces a radial pressure gradient to balance the centrifugal forces,

$\frac{\partial P}{\partial r} \approx \rho \frac{W^{2}}{r}$,

where $W$ is the azimuthal velocity in cylindrical coordinates and $r$ is the radial distance from the center of the jet. Figure 6 shows the time-averaged radial pressure gradient and centrifugal terms, both of which have similar structure and order of magnitude, confirming the relation stated in

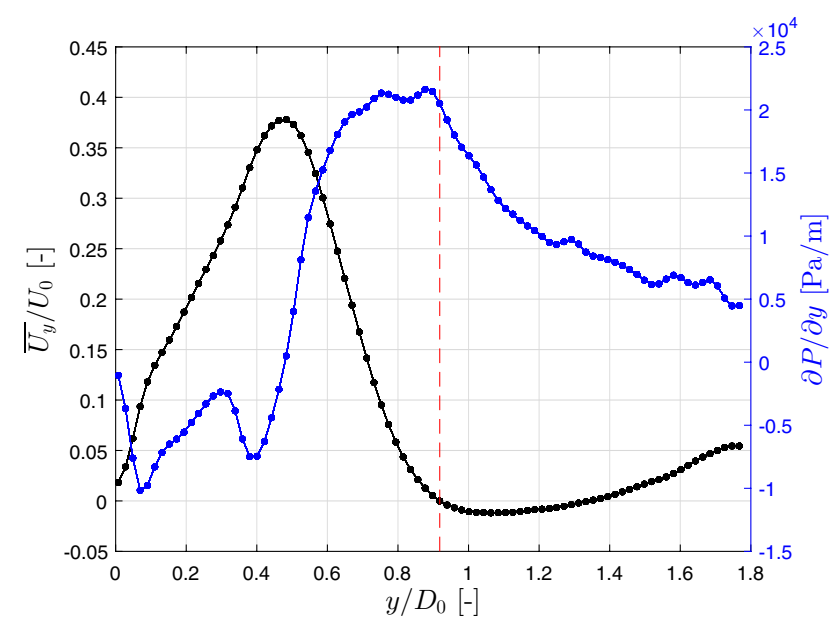

Fig. 7 Axial velocity component $\left(U_{y}\right)$ and pressure gradient $(d P / d y)$ variation along the central axis of the jet $\left(x / D_{0}=z / D_{0}=0\right)$ in the streamwise direction 
Eq. 11. This swirl-induced pressure gradient opens the CRZ and transforms it into a toroidal vortex, as also found in the study by Vanierschot and Van den Bulck (2008a). Fluid is drawn from the sides of the torus to the central axis (Fig. 5a) and the axial velocity is positive along this central axis. Due to the conservation of angular momentum, the fluid moving inward from the sides of the torus increases in tangential velocity near the central axis as shown in Fig. 5c. The increased tangential velocity makes the flow critical and leads to the creation of the second recirculation zone, by a phenomenon referred to as vortex breakdown (LuccaNegro and O'Doherty 2001). Based on the time-averaged axial velocity field in Fig. $5 \mathrm{~b}$ and the variation of the axial flow component in the streamwise direction along the central axis of the jet (Fig. 7, see the red dashed line), the vortex breakdown occurs at $y / D_{o} \approx 0.9$, where axial flow reversal occurs and the positive pressure gradient reaches its maximum value.

The second-order statistics of the flow field are shown in Fig. 8, which reveals that the Reynolds stresses in the flow field are highly anisotropic. Particularly near the CRZ and vortex breakdown bubble, regions of intense mixing occur. Especially, the normal stresses in the shear layer between the vortex breakdown bubble and the jet are high and even larger than the stresses in the outer shear layer between the jet and environment. This feature of vortex breakdown, namely the intense mixing, is very favorable for combustion applications (Gupta et al. 1984).

The time-averaged pressure fields are calculated by either averaging the instantaneous pressure fields over time (Fig. 9a), which has a typical uncertainty of less than $1 \%$ with a $95 \%$ confidence level, or using the Reynolds averaged momentum equation (Fig. 9b). The close correspondence
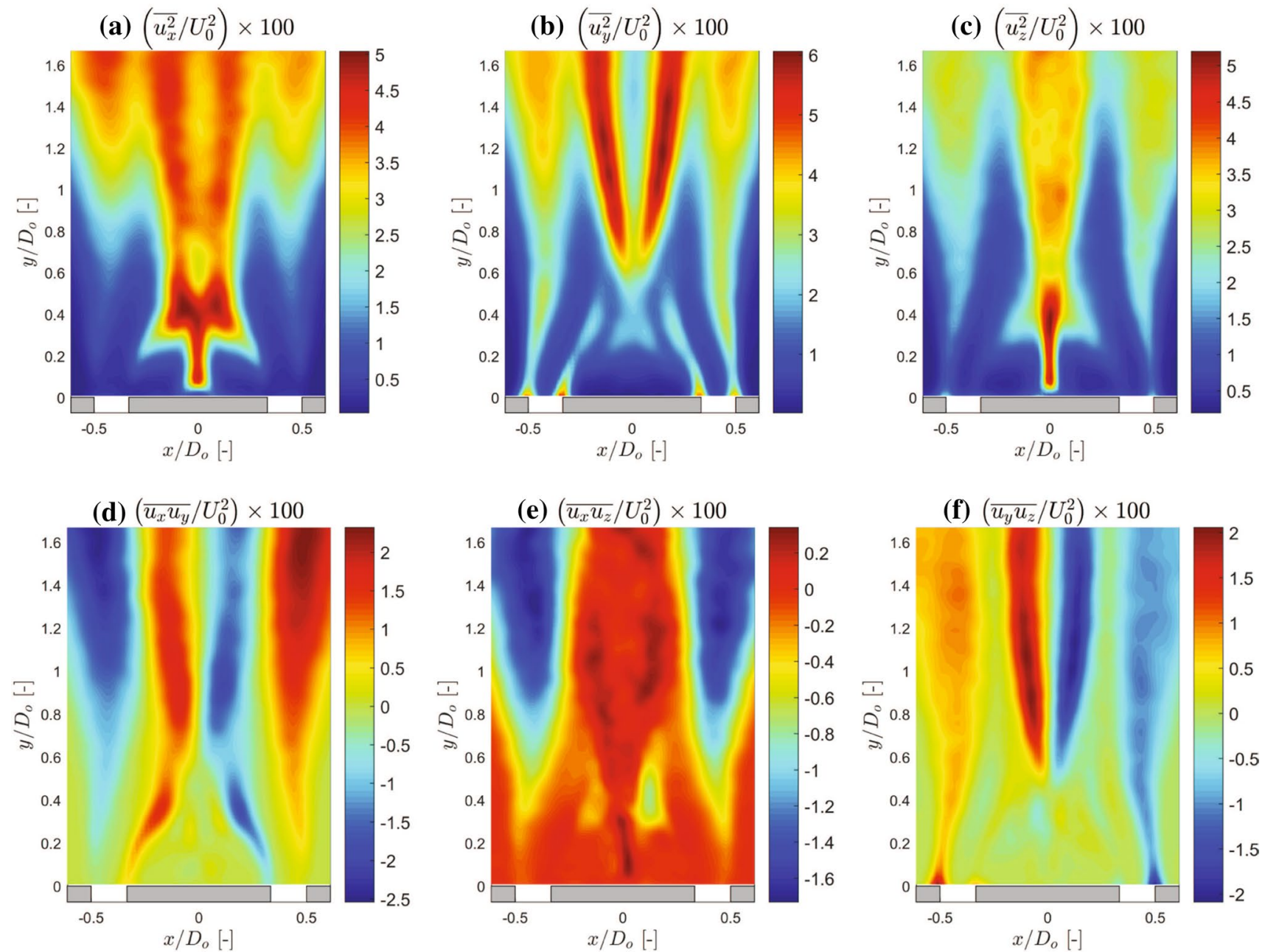

Fig. 8 Second-order statistics of the flow: $\mathbf{a}\left(\overline{u_{x}^{2}} / U_{0}\right) \times 100 ; \mathbf{b}\left(\overline{u_{y}^{2}} / U_{0}\right) \times 100 ; \mathbf{c}\left(\overline{u_{z}^{2}} / U_{0}\right) \times 100 ; \mathbf{d}\left(\overline{u_{x} u_{y}} / U_{0}\right) \times 100 ; \mathbf{e}\left(\overline{u_{x} u_{z}} / U_{0}\right) \times 100 ; \mathbf{f}$ $\left(\overline{u_{y} u_{z}} / U_{0}\right) \times 100$ 


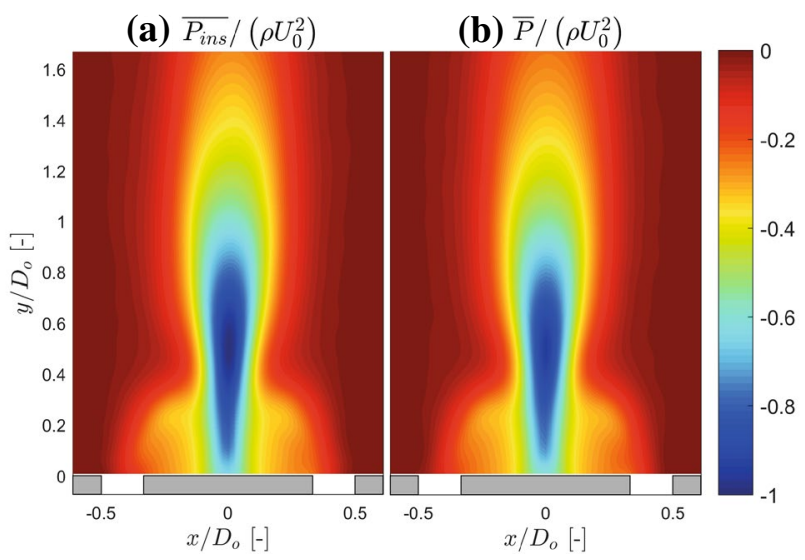

(c)

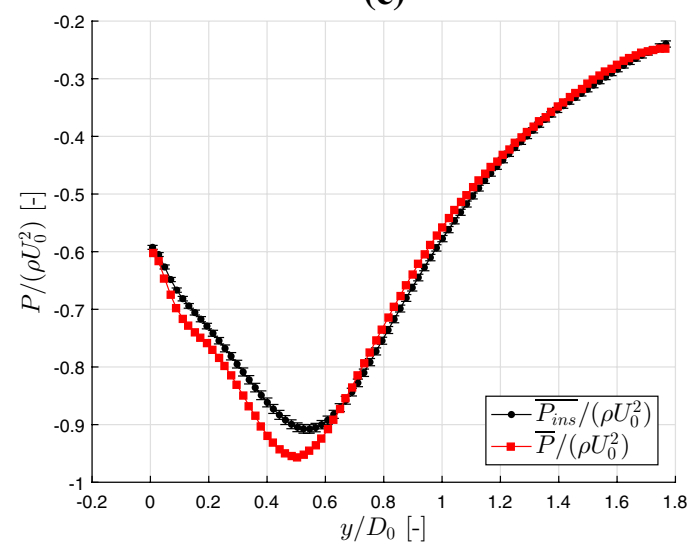

Fig. 9 Normalized time-averaged static pressure fields $\left(P / \rho U_{0}^{2}\right)$ of the annular swirling jet obtained by a averaging the instantaneous pressure fields which are calculated by use of Eq. 2 and $\mathbf{b}$ using the Reynolds-averaged momentum equation (Eq. 3). c Time-averaged normalized static pressure variations along the central axis of the jet $\left(x / D_{0}=z / D_{0}=0\right)$ in the streamwise direction acquired by using the two mentioned methods.

between the two pressure fields (maximum relative difference of $5.2 \%$, see Fig. $9 \mathrm{c}$ ) verifies the statistical convergence of the results as well as the validity of both approaches. The disparity in the region from $y / D_{0}=0.3$ to 0.6 can be attributed to higher Re stress levels (which might require more samples to fully converge) and mostly rotation dominated flow (which may result in larger errors in particle trajectory reconstruction). The pressure field reveals the existence of a low-pressure region along the central axis of the jet. This large region is associated with the CRZ and especially the aforementioned swirl-induced dynamics, as also expressed by Eq. 11 and Fig. 6. Large azimuthal velocities occur particularly downstream of the CRZ (Vanierschot and Van den Bulck 2008a), as can be inferred from the $z$-component of the velocity as shown in Fig. 5c. The azimuthal velocity decreases in the streamwise direction resulting in an increase of pressure along the jet axis (see Fig. 7). This positive pressure gradient in the axial direction leads to the vortex breakdown (Lucca-Negro and O'Doherty 2001).

\subsection{Instantaneous flow structures and pressure fields}

As demonstrated in the previous sections, annular swirling jet flows contain regions of different flow dynamics, which is also evident from the spectral analysis of the $z$ velocity component performed at a number of streamwise locations as shown in Fig. 10. The first distinct region is the CRZ zone $\left(y / D_{0}<0.4\right)$ which is characterized by a peak Strouhal number $\left(\mathrm{St}=f \times D_{h} / U_{0}\right.$ ) of approximately 0.094 (corresponding to a frequency of $9.8 \mathrm{~Hz}$ ). This corresponds to the precession frequency of the CRZ as also reported by Vanierschot et al. (2014). The second region is downstream of the vortex breakdown location $\left(y / D_{0}>0.8\right)$ with a clear peak St of 0.27 , corresponding to a frequency of $28.2 \mathrm{~Hz}$. This is the precessing frequency of the PVC around the central axis, in agreement with what has been also reported by Vanierschot et al. (2016b) based on a novel phase analysis method. For a similar annular jet geometry in a confined configuration, Jones et al. (2012) reported a precession frequency of $\mathrm{St}=0.35$. This relatively high value can be attributed to slightly larger swirl number in their study.

In swirling flow, it is well known that large-scale vortical structures occur (Lucca-Negro and O'Doherty 2001; Syred 2006). Various techniques exist to identify these vortices and in this paper the Q-criterion is applied for this purpose (Jeong and Hussain 1995). This criterion identifies vortices as isosurfaces of positive $Q$. The instantaneous vortical structures of the jet for four subsequent phases during the precession period $\left(T_{\text {pre }}\right)$ are shown in Fig. 11,

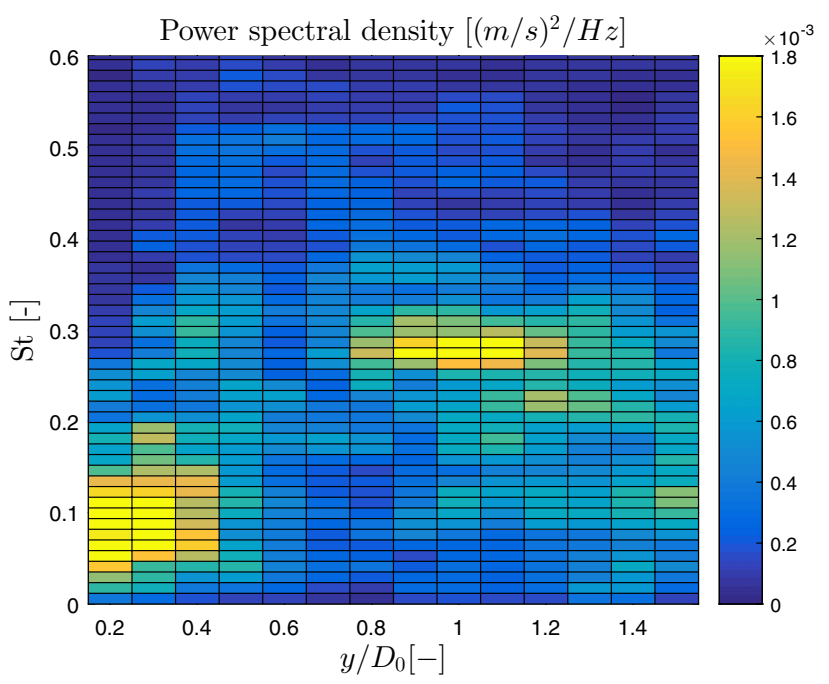

Fig. 10 Power spectral density of the $z$-velocity at several streamwise locations on the central axis of the jet 

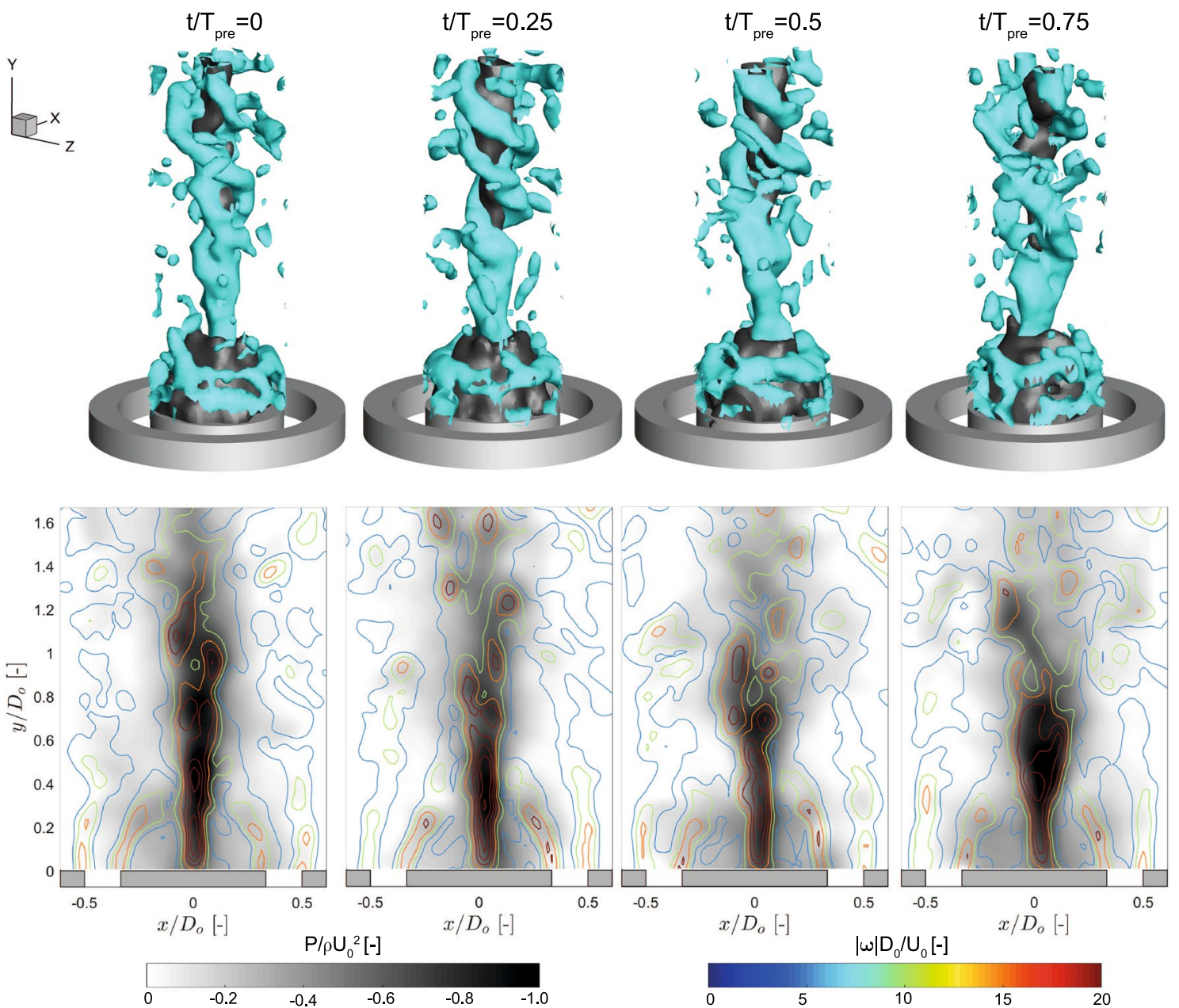

Fig. 11 Instantaneous flow structures of the jet at four phases of the vortex precession period. First row: isosurfaces of $Q /\left(V_{0} / D_{o}\right)^{2}=11.5$ (cyan) and $U_{y} / U_{0}=0$ (gray). Second row:

where isosurfaces of $Q /\left(V_{0} / D_{o}\right)^{2}=11.5$ are shown in cyan. The gray isosurfaces correspond to contours of zero axial velocity, thus indicating the outer contours of the backflow regions. In the second row, instantaneous pressure contours are plotted together with the non-dimensional vorticity magnitude contourlines in the central plane of the jet.

The zero axial velocity isosurfaces clearly display two separate recirculation zones in the flow fields. The first one in the immediate wake of the centerbody and the second zone is located at a more downstream location wrapped around by the helical vortex formation. The former is essentially the toroidal CRZ (Vanierschot and Van den Bulck 2008a) as can be evidenced from the ring-shaped vortical structure. The latter, on the other hand, is located downstream of the vortex core which is aligned along the central axis of the jet in the lower part of the visualized flow region. This vortex core is seen to break up into a precessing vortical instantaneous normalized pressure contours with the non-dimensional vorticity magnitude contourlines plotted in the central plane of the jet

structure downstream, which corresponds to the spiral mode of vortex breakdown (Vanierschot et al. 2016a). This helical structure is wrapped around the breakdown bubble and it is winding in the opposite direction of the swirl, as has been frequently observed also in other studies (Lucca-Negro and O'Doherty 2001). The vortex core and the helical vortex structure result in the formation of low-pressure regions, which is clearly demonstrated by means of the contour plots in the second row in Fig. 11. There is a high correlation between the low-pressure regions and the coherent vortical structures before and after the vortex breakdown point. The lowest mean pressure values and the highest pressure fluctuation levels (see Fig. 12) occur in the central vortex core region upstream of the breakdown location $\left(y / d_{0} \approx 0.5\right)$. This is correlated with the increase of the azimuthal velocity of the fluid being drawn inwards due to radial pressure gradient. 


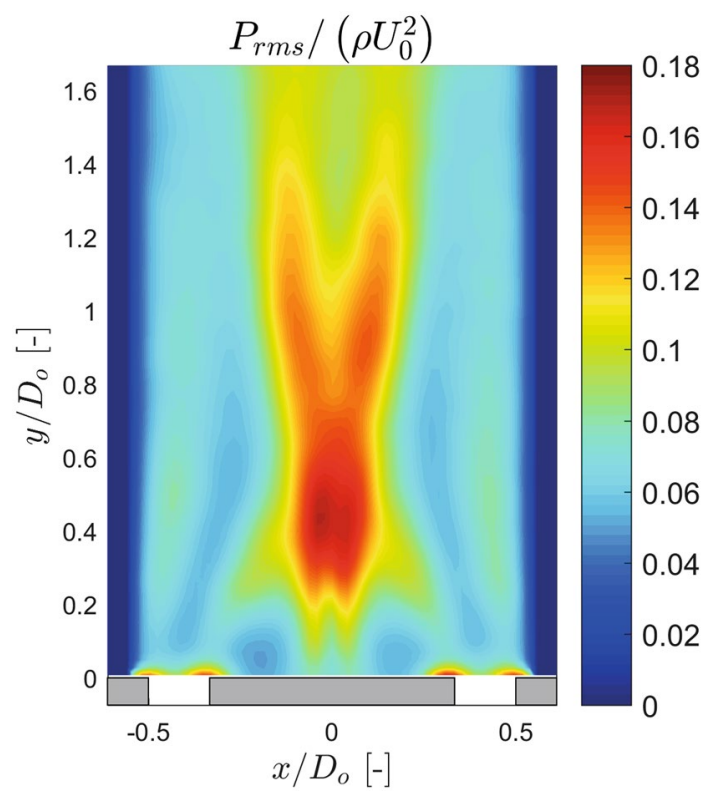

Fig. 12 Contours of normalized pressure fluctuations $\left(P_{r m s} / \rho U_{0}^{2}\right)$ plotted in the central plane of the jet

POD analysis of the instantaneous three-dimensional pressure fields reveal that $50 \%$ of the total energy is captured in the first 60 modes. To assess the dynamics of the modes which have at least $1 \%$ of the total energy, power spectral densities of the time coefficients for the first 20 modes are shown in Fig. 13. It is clear that the first two modes $\left(\mathrm{POD}_{1}\right.$ and $\mathrm{POD}_{2}$ ) share the same peak $\mathrm{St}$ of approximately 0.28 (corresponding to the frequency of $29 \mathrm{~Hz}$ ), which has a high correlation with the precession frequency, while the eighth and ninth modes $\left(\mathrm{POD}_{8}\right.$ and $\left.\mathrm{POD}_{9}\right)$ appear as a second

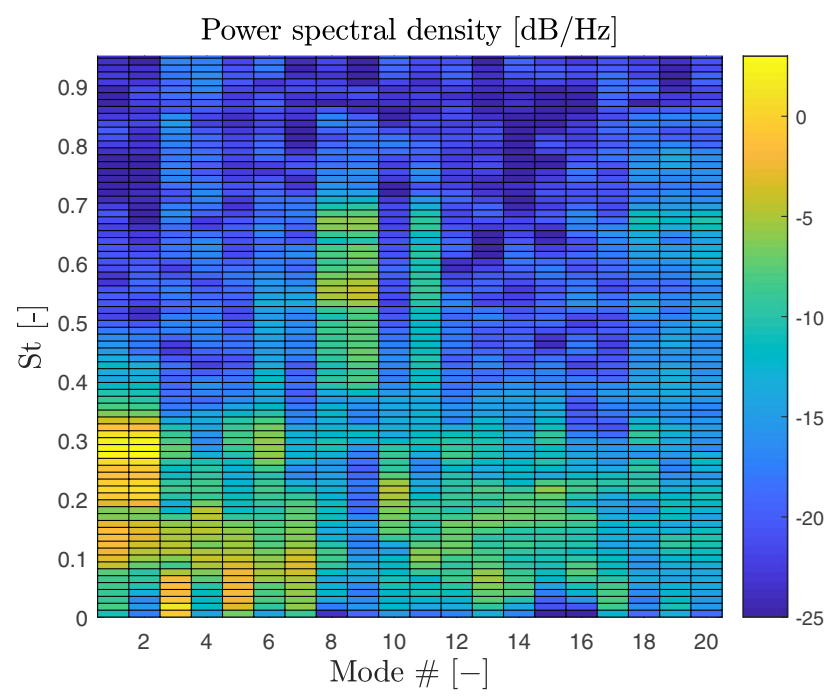

Fig. 13 Power spectral density of the POD time coefficients for the first 20 modes
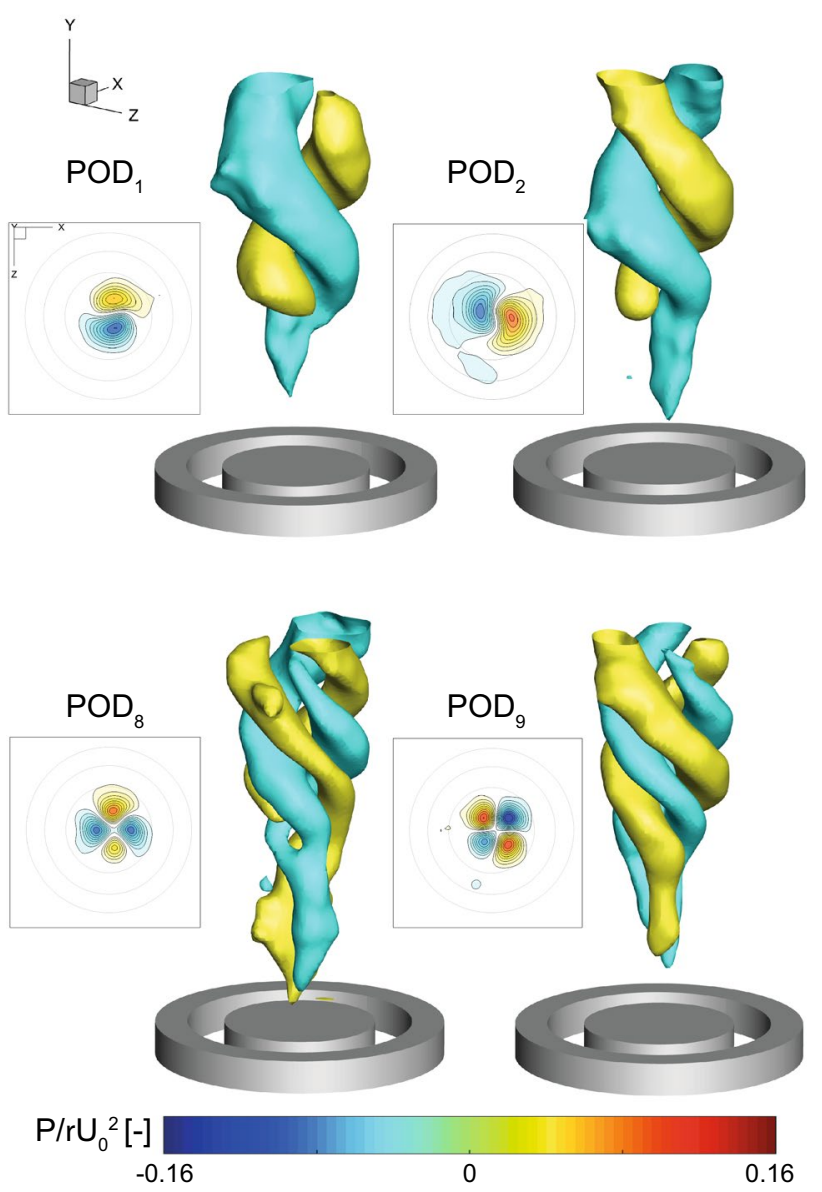

Fig. 14 Representation of four POD modes $\left(\mathrm{POD}_{1}, \mathrm{POD}_{2}, \mathrm{POD}_{8}\right.$ and $\left.\mathrm{POD}_{9}\right)$ by means of isosurfaces of $P /\left(\rho U_{0}^{2}\right)=-0.03$ (cyan) and 0.03 (yellow) complemented with the normalized pressure contour plots in a cross-flow plane $(x-z$ plane $)$ at $y / D_{0}=1$

harmonic of the first two modes with a peak St number of approximately 0.56 (corresponding to the frequency of $58 \mathrm{~Hz}$ ). The pressure fields for these four modes are shown in Fig. 14.

The first two POD modes account for approximately $7.5 \%$ of the total energy $\left(3.85 \%\right.$ for the $\mathrm{POD}_{1}$ and $3.62 \%$ for the $\mathrm{POD}_{2}$ ). The $\mathrm{POD}_{2}$ structures appear as the $\pi / 2$ rotated form of the $\mathrm{POD}_{1}$ structures, as can be evidenced from the cross-flow plane contour plots, while both displaying a helical formation. The phase shift of $\pi / 2$ in the time coefficients (not shown here) confirms that these two modes can be interpreted as the orthogonal components of a precessing structure. Relatively low energy values, even for the first two POD modes, can be attributed to the highly turbulent characteristics of the flow. For the eighth and the ninth modes, on the other hand, the energy is $2.8 \%$ of the total energy $\left(1.43 \%\right.$ for the $\mathrm{POD}_{8}$ and $1.37 \%$ for the $\mathrm{POD}_{9}$ ). The structures for these modes are double-helical formations, which resemble to the vortical structures 
observed in the flow field analysis. The $\mathrm{POD}_{9}$ structures are $\pi / 4$ rotated form of the $\mathrm{POD}_{8}$ structures with a phase shift of $\pi / 2$ between the time-coefficient signals. A loworder flow representation, taking both these two mode pairs into account, results in a precessing helical structure at a St of 0.28 , which suggests that the precessing helical vortex structure is the dominant flow structure in terms of pressure fluctuations.

\section{Conclusions}

In this study, spatial and temporal characteristics of the three-dimensional swirling annular jet flow have been studied using time-resolved tomographic particle image velocimetry technique. The measurements were performed in two modes: (1) a relatively low frequency double-frame mode to increase the measurement time and achieve a converged statistical analysis; (2) a high frequency single-frame mode to enable visualization of the time-series phenomenon. In addition to the statistical and temporal analysis of the threedimensional velocity fields, both time-averaged and instantaneous pressure fields were calculated from the velocity data by use of the governing flow equations. The results are presented together with a comprehensive analysis on the accuracy of the measurements. In this respect, this study serves for revealing the three-dimensional characteristics of swirling annular jet flows and the associated pressure fields.

Time-averaged results reveal two distinct flow structures. The first one is a toroidal central recirculation zone behind the centerbody. This torus is created by a swirl-induced radial pressure gradient which balances the centrifugal forces. Further downstream, the conservation of tangential momentum creates regions of high tangential velocities. The tangential velocities decrease in the downstream direction resulting in an increase of pressure along the jet axis, i.e. generating a positive pressure gradient and leading to the vortex breakdown.

The visualization of the instantaneous flow reveals that the central vortex core, which is correlated with the lowpressure region and high-pressure fluctuation levels on the central axis, breaks up into a precessing vortex core, which is wrapped around the breakdown bubble in the opposite direction of swirl. This structure precesses around the central axis at a Strouhal number of 0.27 in the swirl direction, which is also supported by the proper orthogonal decomposition of the instantaneous pressure fields. The POD analysis suggests that the precessing helical vortex structure, which forms as a result of the vortex breakdown, is the most dominant flow structure as far as the pressure fluctuations are concerned.

Acknowledgements The authors would like to thank the Flemish Fund for Scientific Research FWO-Vlaanderen and the J.M.
Burgerscentrum for their financial support of the measurement campaign.

Open Access This article is distributed under the terms of the Creative Commons Attribution 4.0 International License (http://creativecommons.org/licenses/by/4.0/), which permits unrestricted use, distribution, and reproduction in any medium, provided you give appropriate credit to the original author(s) and the source, provide a link to the Creative Commons license, and indicate if changes were made.

\section{References}

Al-Abdeli YM, Masri AR (2004) Precession and recirculation in turbulent swirling isothermal jets. Combust Sci Technol 176(5-6):645-665

Alekseenko SV, Bilsky AV, Dulin VM, Markovich DM (2007) Experimental study of an impinging jet with different swirl rates. Int J Heat Fluid Flow 28(6):1340-1359

Atkinson C, Soria J (2009) An efficient simultaneous reconstruction technique for tomographic particle image velocimetry. Exp Fluids 47(4-5):553

Atkinson C, Coudert S, Foucaut JM, Stanislas M, Soria J (2011) The accuracy of tomographic particle image velocimetry for measurements of a turbulent boundary layer. Exp Fluids 50(4):1031-1056

Beér JM, Chigier NA (1972) Combustion aerodynamics. Wiley, New York

Benedict L, Gould R (1996) Towards better uncertainty estimates for turbulence statistics. Exp Fluids 22(2):129-136

Billant P, Chomaz JM, Huerre P (1998) Experimental study of vortex breakdown in swirling jets. J Fluid Mech 376:183-219

Cala C, Fernandes E, Heitor M, Shtork S (2006) Coherent structures in unsteady swirling jet flow. Exp Fluids 40(2):267-276

Chterev I, Foley C, Foti D, Kostka S, Caswell A, Jiang N, Lynch A, Noble D, Menon S, Seitzman J et al (2014) Flame and flow topologies in an annular swirling flow. Combust Sci Technol 186(8):1041-1074

Chterev I, Sundararajan G, Emerson B, Seitzman J, Lieuwen T (2017) Precession effects on the relationship between time-averaged and instantaneous reacting flow characteristics. Combust Sci Technol 189(2):248-265

Dugué J, Weber R (1992a) Design and calibration of a $30 \mathrm{~kW}$ natural gas burner for the University of Michigan. In: Tech. Rep. C74/y/1, International Flame Research Foundation, Ijmuiden, The Netherlands

Dugué J, Weber R (1992b) Design and calibration of a $30 \mathrm{~kW}$ natural gas burner for the University of Michigan. In: Tech. Rep. C74/y/5, International Flame Research Foundation, Ijmuiden, The Netherlands

Elsinga GE, Scarano F, Wieneke B, van Oudheusden BW (2006) Tomographic particle image velocimetry. Exp Fluids 41(6):933-947

García-Villalba M, Fröhlich J (2006) LES of a free annular swirling jet-dependence of coherent structures on a pilot jet and the level of swirl. Int J Heat Fluid Flow 27(5):911-923

García-Villalba M, Fröhlich J, Rodi W (2006) Identification and analysis of coherent structures in the near field of a turbulent unconfined annular swirling jet using large eddy simulation. Phys Fluids 18(5):055103

Ghani A, Poinsot T, Gicquel L, Staffelbach G (2015) LES of longitudinal and transverse self-excited combustion instabilities in a bluff-body stabilized turbulent premixed flame. Combust Flame 162(11):4075-4083 
Gupta A, Lilley D, Syred N (1984) Swirl flows. Abacus Press, Tunbridge Wells

Jeong J, Hussain F (1995) On the identification of a vortex. J Fluid Mech 285:69-94

Jones W, Lyra S, Navarro-Martinez S (2012) Large eddy simulation of turbulent confined highly swirling annular flows. Flow Turbul Combust pp 1-24

Litvinov IV, Shtork SI, Kuibin PA, Alekseenko SV, Hanjalic K (2013) Experimental study and analytical reconstruction of precessing vortex in a tangential swirler. Int J Heat Fluid Flow 42:251-264

Lucca-Negro O, O’Doherty T (2001) Vortex breakdown: a review. Prog Energ Combust 27(4):431-481

Markovich D, Abdurakipov S, Chikishev L, Dulin V, Hanjalić K (2014) Comparative analysis of low-and high-swirl confined flames and jets by proper orthogonal and dynamic mode decompositions. Phys Fluids 26(6):065109

Martinelli F, Cozzi F, Coghe A (2012) Phase-locked analysis of velocity fluctuations in a turbulent free swirling jet after vortex breakdown. Exp Fluids 53(2):437-449

Oberleithner K, Sieber M, Nayeri C, Paschereit C, Petz C, Hege HC, Noack B, Wygnanski I (2011) Three-dimensional coherent structures in a swirling jet undergoing vortex breakdown: stability analysis and empirical mode construction. J Fluid Mech 679:383-414

Oberleithner K, Paschereit C, Wygnanski I (2014) On the impact of swirl on the growth of coherent structures. J Fluid Mech 741:156-199

Oberleithner K, Stöhr M, Im SH, Arndt CM, Steinberg AM (2015) Formation and flame-induced suppression of the precessing vortex core in a swirl combustor: experiments and linear stability analysis. Combust Flame 162(8):3100-3114

Panda J, McLaughlin D (1994) Experiments on the instabilities of a swirling jet. Phys Fluids 6(1):263-276

Percin M, Van Oudheusden B (2015) Three-dimensional flow structures and unsteady forces on pitching and surging revolving flat plates. Exp Fluids 56(2):1-19

Pröbsting S, Scarano F, Bernardini M, Pirozzoli S (2013) On the estimation of wall pressure coherence using time-resolved tomographic PIV. Exp Fluids 54(7):1567

Reichel TG, Terhaar S, Paschereit O (2015) Increasing flashback resistance in lean premixed swirl-stabilized hydrogen combustion by axial air injection. J Eng Gas Turbines Power 137(7):071503

Scarano F (2012) Tomographic PIV: principles and practice. Meas Sci Technol 24(1):012001

Scarano F, Elsinga GE, Bocci E, van Oudheusden BW (2006) Investigation of 3-D coherent structures in the turbulent cylinder wake using Tomo-PIV. In: 13th international symposium on applications of laser techniques to fluid mechanics, Lisbon, Portugal, vol 20

Sciacchitano A, Wieneke B (2016) PIV uncertainty propagation. Meas Sci Technol 27(8):084006

Sheen H, Chen W, Jeng S (1996) Recirculation zones of unconfined and confined annular swirling jets. AIAA J 34(3):572-579

Sirovich L (1987) Turbulence and the dynamics of coherent structures. i. coherent structures. Q Appl Math 45(3):561-571

Syred N (2006) A review of oscillation mechanisms and the role of the precessing vortex core (PVC) in swirl combustion systems. Prog Energy Combust Sci 32(2):93-161

van Oudheusden BW (2013) PIV-based pressure measurement. Meas Sci Technol 24(3):032001

Vanierschot M, Van den Bulck E (2008a) Influence of swirl on the initial merging zone of a turbulent annular jet. Phys Fluids 20(10): 105104

Vanierschot M, Van den Bulck E (2008b) Planar pressure field determination in the initial merging zone of an annular swirling jet based on stereo-PIV measurements. Sensors 8(12):7596-7608

Vanierschot M, Van den Bulck E (2011) Experimental study of low precessing frequencies in the wake of a turbulent annular jet. Exp Fluids 50(1):189-200

Vanierschot M, Van Dyck K, Sas P, Van den Bulck E (2014) Symmetry breaking and vortex precession in low-swirling annular jets. Phys Fluids 26(10):105-110

Vanierschot M, Percin M, van Oudheusden B (2016a) Visualization of the structure of vortex breakdown in free swirling jet flow. In: 18th international symposium on the application of laser and imaging techniques to fluid mechanics, Lisbon, Portugal

Vanierschot M, Percin M, Van Oudheusden B (2016b) A novel phaseaveraging method based on vortical structure correlation. In: International workshop on non-intrusive optical flow diagnostics

Warda H, Kassab S, Elshorbagy K, Elsaadawy E (1999) An experimental investigation of the near-field region of free turbulent round central and annular jets. Flow Meas Instrum 10(1):1-14

Wegner B, Maltsev A, Schneider C, Sadiki A, Dreizler A, Janicka J (2004) Assessment of unsteady RANS in predicting swirl flow instability based on LES and experiments. Int J Heat Fluid Flow 25(3):528-536

Westerweel J, Scarano F (2005) Universal outlier detection for PIV data. Exp Fluids 39(6): 1096-1100

Wieneke B (2008) Volume self-calibration for 3D particle image velocimetry. Exp Fluids 45(4):549-556

Willert CE, Gharib M (1991) Digital particle image velocimetry. Exp Fluids 10(4):181-193 